\title{
APUNTES PARA FUNDAMENTAR UN PROYECTO ÉTICO-POLÍTICO PROFESIONAL EN PUERTO RICO
}

\section{Jesús M. Cabrera Cirilo}

"Ya hemos dicho y afirmado que siempre ha habido miseria. Pero no siempre ha habido cuestión social. La cuestión social ha sido producida por la misma intelectualidad, es decir, ha nacido con los mismos adelantos científicos de la industria y de la agricultura"

R. del Romeral (1904) ${ }^{2}$

A Blanca Canales e Isabel Rosado, fuentes de inspiración para las nuevas generaciones de trabajadores y trabajadoras sociales puertorriqueños.

${ }^{1}$ Trabajador Social en el Departamento de Justicia.

${ }^{2}$ Seudónimo utilizado por Ramón Romero Rosa quien desarrolló la obra; La cuestión social y Puerto Rico publicada en San Juan en el año 1904. El trabajo de Romeral demuestra que la discusión de la "cuestión social" no fue, ni es actual en Puerto Rico, y que también su comprensión es la apropiación de la categoría. Su entendido es a todas luces, el sentido que queremos aprehender. 


\section{Resumen}

El objetivo principal de este escrito es discutir asuntos medulares de la profesión para finalmente recomendar las alternativas que pueden ayudar a adelantar la renovación del Trabajo Social puertorriqueño, creando un nuevo proyecto ético-político. Pero antes corresponde dar el primer paso. El más importante es romper con todo ese imaginario creado por más de 80 años de reproducción ideológica. Así las cosas, la naturaleza del ethos puertorriqueño se constituye de una amalgama de contradicciones y mediaciones destacándose el conservadurismo propio de la perspectiva religiosa (católicaprotestante). En este sentido, la parte ética nace conjuntamente con su génesis puesto que es su fundamento ideológico y filosófico lo que le otorga las posibilidades y limitaciones de su quehacer —un cierto modo de comportamiento - y generaliza la cohesión del colectivo. Sobre está limitación filosófica e ideológica es que hay que pasar revisión para poder llegar a la adultez del Trabajo Social puertorriqueño.

\section{Abstract}

The principal objective of this article is to discuss key issues for the profession of social work. Recommendations will be presented that may help to move forward the renewal of a Puerto Rican Social Work, and create a new ethical political project. First, the article will address to the decomposition of the reproduction of the ideological imaginary created eighty years ago. The nature of the Puerto Rican ethos is constituted by a mixed of contradictions and mediations, standing out the conservatism proper of a religious perspective (catholic-protestant). In this sense, the ethics arise jointly with the genesis that is the ideological and philosophical foundation. This foundation gives the possibilities and limitations for the work - a certain mode of behavior - and the collective unity. This ideological and philosophical limitation should be revised in order to achieve adulthood of the Puerto Rican Social Work.

\section{Nota introductoria}

Son variados los esfuerzos por otorgarle mayor criticidad a la educación en Trabajo Social en Puerto Rico. Al menos así se ve reflejado en la Escuela Graduada Beatriz Lasalle; especialmente en el protagonismo de la facultad y de sus estudiantes en la huelga universitaria, entre muchas otras acciones. Además, dentro del Colegio de Profesionales del Trabajo Social hay intentos de cambios. Aunque, separadamente, ambas situaciones tienen el mismo norte: levantar el Trabajo Social nacional concretando un proyecto éticopolítico. ${ }^{3}$ Todo lo que está sucediendo demuestra que realmente nos preocupa debatir lo relacionado con la formación y práctica cotidiana. En este sentido se está iniciando el primer paso, quizás el más difícil de todos.

El objetivo principal de este escrito es discutir asuntos medulares de la profesión para finalmente recomendar las alternativas que pueden ayudar a adelantar la renovación del Trabajo Social puertorriqueño, creando un nuevo proyecto ético-político.

Por consiguiente, es importante y necesario aportar a dichos Es indudable el trabajo que se ha estado desarrollando en esta dirección en el Colegio desde el 2008. La iniciativa de un grupo comprometido con este proyecto llevó a discutir este tema en las diferentes regiones concretando varios documentos entre estos, una sistematización de las reuniones. Se recomienda la lectura de Cuaderno de Trabajo Proyecto Ético-Político Profesional, Reuniones Regionales. 
esfuerzos. Es indiscutible que sólo se podrán lograr todos los objetivos, mediatos e inmediatos, ampliando e incluyendo a más personas al debate. En este artículo pretendemos ofrecer algunos planteamientos que, a nuestro entender, debe contener el mismo, no sin antes repasar los fundamentos originales del Trabajo Social puertorriqueño.

\section{Síntesis de la naturaleza de la ética profesional en Puerto Rico.}

En términos generales-universales, el Trabajo Social es una profesión que nace en un momento específico del desarrollo del capitalismo: el monopólico (Netto, 1997) cuando por diferentes situaciones el Estado opta por intervenir en las relaciones sociales. Por esto es fundamental que entendamos que el Trabajo Social se concatena con muchas contradicciones. Por un lado somos trabajadoras y trabajadores asalariados que fungimos en la reproducción de las relaciones sociales en el capitalismo. Por otro, indiscutiblemente aportamos y apoyamos a un segmento de la población marginada proveyéndole oportunidades para su desarrollo y, en muchos casos, ayudamos a defender sus derechos, entre muchas otras funciones importantes. Ahora bien, Barroco (2004), asegura que la naturaleza de la ética profesional es:

Un modo particular de objetivación de la vida ética. Sus particularidades, se inscriben en la relación entre el conjunto complejo de necesidades que legitiman la profesión en la división sociotécnica del trabajo confiriéndole determinadas demandas y sus respuestas específicas, entendidas en su dimensión teleológica y en razón de las implicaciones éticopolíticas del producto concreto de su acción [...]. En este sentido, el ethos profesional es un modo de ser constituido en la relación compleja entre las necesidades socioeconómicas e ideoculturales y las posibilidades de elección introducidas en las acciones ético-morales, lo que apunta para su diversidad, cambiabilidad y contradicción (Barroco, 2004: 84).

Si es así, la ética profesional es fundamentalmente un conjunto de actividades y acciones que responden a las exigencias de la realidad concretándose como respuestas a las mismas. Es a fin de cuentas un cierto comportamiento ante variadas situaciones y exigencias en el quehacer cotidiano de ese "ser profesional". E ethos del Trabajo Social es una construcción histórica que se sostiene en un conjunto de esquemas valorativos ideológicos, filosóficos, culturales, teóricos y, en muchas ocasiones, antagónicos. Por ello, la ética en el Trabajo Social es mucho más que un Código de Ética. Este Código es un esfuerzo que representa y aglutina todo lo anterior para ofrecer opciones para dicho comportamiento en ciertas coyunturas, junto con todas las acciones correctivas y sanciones cuando no se cumple con los parámetros establecidos por el gremio. Es de aclarar que, al igual que todo lo social, lo ético también es cambiante, dinámico. Es decir, se transforma en tanto y en cuanto lo hacen los proyectos sociales más amplios, adaptándose a dichos requerimientos. Entendido esto, la pregunta obligatoria sería ¿cuá es la naturaleza del ethos del Trabajo Social en Puerto Rico?

En primer lugar, con la invasión norteamericana a la Isla se cambió una estructura colonialista por otra. Los norteamericanos destruyen la lógica colonialista decadente anterior y con ello la esfera del trabajo, la cotidianidad isleña, los esfuerzo por la transculturación y las relaciones sociales. Empero, no es hasta que el Estado (en su 
característica imperialista-colonialista estadounidense) reconstruye las instituciones gubernamentales destruidas, desarticuladas o simplemente sustituidas a la "americana" que se abre el espacio para la incursión del Trabajo Social en Puerto Rico.

Es con las reconfiguraciones de las estructuras coloniales y su consolidación por medio de las leyes "orgánicas" (1900 y 1917), que se forjan los espacios laborales que legitimarán las profesiones, sus quehaceres y las sanciones legales relacionadas con las funciones. Pero no de forma inmediata, ni automática. Es sólo cuando el Estado, en este caso imperialista-colonial, se adjudica en el capitalismo monopólico la prerrogativa de tomar partido en "la cuestión social colonial" apropiándose de la política social privada ejecutada, principalmente, por La Cruz Roja Americana, capítulo de Puerto Rico ( Cabrera, 2010).

Con las referidas leyes "orgánicas" se adjudica, confirma y condiciona la perpetuidad de la dominación de Estados Unidos sobre el territorio y sus pobladores, pero es con la última (1917), que se instauran instrumentos jurídicos, instituciones y estructuras de algún tipo de gobierno para atender los antagonismos de la "cuestión social colonial"4 en ciernes. Al fundarse la entidad

${ }^{4}$ La "cuestión social colonial" es una categoría de análisis para especificar "la cuestión social" en el contexto colonial. Intenta modular dos situaciones que median las condiciones socio-históricas para la génesis profesional en Puerto Rico. Por un lado, la llamada "cuestión nacional", que abarca las luchas y confrontaciones de un sector del pueblo puertorriqueño contra la invasión imperialista estadounidense. En otras palabras, la pugna por la independencia política y económica; por un Estado soberano. Además, comprende la sujeción de que es objeto la población, e incluye la conexión dialéctica colonizado-colonizador. Por otro, las contradicciones capital-trabajo propias de la imposición del modo de producción capitalista yanqui, en un país con una economía agraria y atrasada tecnológicamente. También incluye las luchas de la clase obrera en esta subordinación colonial y las posiciones de los líderes obreros en cuanto a estos dos aspectos; la atadura colonial y el capital-trabajo. institucional se abre la posibilidad de la incursión de la profesión del Trabajo Social en el panorama puertorriqueño.

Con la invasión llegan muchos cambios que no se limitaron a la esfera político-económica. Se vio afectado también el religioso-cultural; la protestantización de las esferas socioculturales aparece como otro aditamento de agresión. La cultura religiosa puertorriqueña estuvo determinada por el sincretismı entre el catolicismo centenario y los rituales africano-caribeños sustentados en los cuatrocientos años de colonización española y de la producción cimentada en la mano de obra esclava negra. Esto hace que la ritualidad caribeña sea especial y muy internalizada y arraigada sociológicamente, por lo que las personas que se iniciaron en la formación del Trabajo Social no eran ni fueron protestantes. La naturaleza del Trabajo Social puertorriqueño y su ética están transversalmente intervenidas por la teología cristiano-católica. No significa que la profesión haya estado determinada por el catolicismo como en otras latitudes, o que haya sido la Iglesia Católica la instauradora de la categoría profesional, sino que se inició como una carrera secular, pero que las personas que se inscribieron eran católicas. Lo que no descarta, que el judeo-cristianismo Apostólico Romano haya motivado a diferentes personalidades a inscribirse en una carrera que hacía trabajo con los pobres; en otras palabras, que ayudaba a los más necesitados. Esta condensación religiosocultural es lo que diferencia el Trabajo Social puertorriqueño de muchos otros, especialmente latinoamericanos, donde los proyectos profesionales fueron establecidos por la Iglesia Católica. ${ }^{5}$

\footnotetext{
${ }^{5}$ Ver: Iamamoto (1997).
} 
Así las cosas, la naturaleza del ethos puertorriqueño se constituye de una amalgama de contradicciones y mediaciones destacándose el conservadurismo propio de la perspectiva religiosa (católica-protestante). Esta visión de mundo provee una lectura de la realidad que resulta inmutable, naturalizada y desprendida del cambio, pues todo ya está determinado por fuerzas más allá de la voluntad humana, fuerzas superiores que trazan el porvenir de la sociedad. Evidente y claramente se trata de una combinación entre el positivismo ${ }^{6}$ y la teología tomista ${ }^{7}$ (Barroco, 2004). Ante esto, lo que le queda a la intervención del trabajador y la trabajadora social es, simple y llanamente, ayudar a mejorar su condición, a su adaptación o a su resignación a lo ya dado, lo establecido, lo real. En este sentido, la parte ética nace conjuntamente con su génesis puesto que es su fundamento ideológico y filosófico lo que le otorga las posibilidades y limitaciones de su quehacer - un cierto modo de comportamiento - y generaliza la cohesión del colectivo. Sobre está limitación filosófica e ideológica es que hay que pasar revisión para poder llegar a la adultez del Trabajo Social puertorriqueño. Para lograrlo, es necesario comprender la labor que ejecuta el Trabajo Social en la reproducción de las relaciones sociales en el capitalismo.

Para finalizar, no se puede negar que en algunas ocasiones y momentos históricos se han hecho verdaderos intentos dirigidos a

${ }^{6} \mathrm{El}$ positivismo ha sido una de las corrientes más influyentes de finales del siglo XIX y principios del XX. Se entiende como un sistema filosófico basado en la experiencia y el conocimiento empírico de los fenómenos naturales, en el cual la Metafísica y la Teología son sistemas de conocimientos imperfectos e inadecuados. El desarrollo de la ciencia en los últimos siglos le ha permitido pusicionarse como una disciplina del "verdadero conocimiento", ya que lo aceptado y creíble es primordialmente lo que se puede comprobar por algún proceso positivo (Cabrera, 2010). Se refiere a toda la producción teológica de Santo Tomás de Aquino. ofrecerle una nueva perspectiva a la profesión. Sin embargo, estos no han calado profundas raíces. Los mismos han sido muy puntuales y no determinantes.

Es indiscutible que el conservadurismo es el proyecto que ha dominado hegemónicamente la carrera. ${ }^{8}$

\section{Elementos fundamentales para la creación de un proyecto ético- político en Puerto Rico.}

Para comenzar, ¿por qué ético-político y no solamente ético? Es bastante sencilla la contestación; porque en esta ocasión se debe superar y transgredir la supuesta neutralidad de la profesión, tomando partido en las decisiones y luchas sociales. En esta oportunidad habrá que hacer todo lo contrario; desde un posicionamiento ético denunciar y combatir todo lo opresivo, sobre todo, el capitalismo. En palabras más sencillas, los trabajadores y las trabajadoras sociales deben convertirse en entes politizados.

Pero antes corresponde dar el primer paso. El más importante es romper con todo ese imaginario creado por más de 80 años de reproducción ideológica. Hay dos concepciones que son primordiales desmitificar. Uno ya ha sido mencionado, la supuesta neutralidad del quehacer profesional. El otro es la perspectiva de que el Trabajo Social promueve el "cambio social" y " la justicia social". Será analizado el primer asunto, el concepto de que la labor

\begin{abstract}
Así lo explica Seda en entrevista realizada por el autor el domingo 1 de marzo de 2009, en Mayagüez, Puerto Rico.; "Claro, porque todavía impera esa mentalidad en este país, en términos generales. Aquí no hay una educación liberadora [...] ya hemos hablado de una de ellas, la educación a la que hemos estado expuestos. Los profesores de Trabajo Social, que se supone que somos los que llevamos ese mensaje diferente a los estudiantes lo hemos internalizado muy pocas personas... muy pocas personas... [...] nuestra formación ha sido una bien conservadora y a pesar de que en las últimas décadas se ha estado estudiando y trayendo escritos como este, son muy pocos y no se ha internalizado eso, en las mayorías de las Escuelas y en los gremios profesionales".
\end{abstract}

VOCES DESDE EL TRABAJO SOCIAL

NÚM.1 
es "neutra". En otras palabras, que se sustenta por la objetividad y la imparcialidad. No hay mejor ejemplo, que la manera como es expresado el asunto en el Código de Ética que ha dirigido el destino de muchas generaciones:

El Trabajador Social debe estar alerta y hacer frente a las posibles influencias y presiones ajenas a su función que puedan interferir con el ejercicio de su discreción profesional y la objetividad esencial a su quehacer (Código de Ética: 2).

La Carta Magna es evidencia de lo que se viene discutiendo. Según ésta, los y las profesionales deben estar atentos a toda aquella influencia que pueda "alterar" la supuesta objetividad, la cual es fundamental en su labor. Pero esto es una falsedad; el diario vivir en las instituciones en las cuales se desempeñan, tanto privadas como públicas, es una total contradicción pues la realidad es otra. Los trabajadores y las trabajadoras sociales realmente hacen lo que el patrono que los contrata les pide, sea de prevención (Educación) o punitivo (Justicia, Familia, Corrección). Más aún cuando en algunos espacios laborales ni siquiera hay una real libertad de acción, de intervención ni pericial estando siempre subordinados a otras profesiones. ${ }^{9}$

${ }^{9}$ Esta discusión es muy importante para la profesión. Desde sus inicios el Trabajo Social en Puerto Rico ha mantenido un papel de supeditación a otras profesiones. En los proyectos gubernamentales lo social es un medio para lograr otros objetivos, políticos, económicos, de subordinación, lo que hace del Trabajo Social un "complemento" de otras ramas. Así explica esta situación Seda (1990); "Históricamente, el trabajo social ha sido una profesión subordinada. En el campo de la salud el trabajador social está subordinado al médico, en la educación al maestro, en la vivienda al ingeniero, en la administración de la justicia al abogado, entre otros campos. Se ha internalizado hasta tal punto que el trabajo social es una profesión "auxiliar" a estas otras, que es penoso observar la impotencia autoimpuesta de un gran número de colegas. Y nos preguntamos, ¿qué ha ocurrido en la formación de estos profesionales que no pueden defender con seguridad y valentía su identidad y su competencia profesional?" (Seda, 1990:13).
Ciertamente, nada de objetiva, ni neutral, ni imparcial, sino todo lo contrario, se funciona a partir de las posibilidades dentro de las alternativas que les ofrecen; inclinando la balanza a favor de los financiadores de los programas o proyectos. En palabras sencillas, el Trabajo Social responde a quien hace la política social y bajo el neoliberalismo, ${ }^{10}$ dicha política cada vez es más precaria y atomizada.

Sumado a esto, esta conceptualización deja de lado la subjetividad y la percepción de cada profesional, como si fuera posible desprenderse de todo el bagaje que trae consigo, basada en su experiencia personal e intelectual. En resumidas cuentas, como si fuera posible actuar como máquinas, autómatas, robots que no sienten y que, peor aún, no pueden (o ¿no deben?) tomar decisiones. En la sociedad nada es estático; todo tiene movimiento; por lo tanto, toda acción en ella se constituye en una acción política; en algunos casos para sostener lo establecido y en otros para cambiarlo. El ejercicio profesional está inclinado políticamente hacia uno de los lados; el status quo. Sin embargo, esto no necesariamente tiene que continuar así.

Toda esta visualización de "neutralidad" u "objetividad" es un costal que arrastra el Trabajo Social isleño desde su génesis y que viene atada a la corriente filosófica que le dio vida: el positivismo. Aunque, por supuesto, no es exclusivo del mismo; también se

${ }^{10}$ El neoliberalismo surge como salida a la crisis capitalista mundial. Por ejemplo, se distingue por la transferencia de las responsabilidades del Estado al sector privado, convirtiendo todo lo social en mercancía por la cual todos y todas tenemos que pagar. Entre las más destacadas soluciones se sobresalen; la desregulación financiera, el desmantelamiento de los derechos sociales universales y de las leyes laborales, entre otros.

VOCES DESDE EL TRABAJO SOCIAL

NÚM.1 
entrelaza con las propias particularidades de la construcción profesional en Puerto Rico basada en muchas mediaciones y determinaciones que la constituyeron.

El segundo punto, el cual es irremediablemente inseparable del anterior. Si el ejercicio no es "neutro” entonces ¿por qué seguir insistiendo en la mesiánica visión de que las y los trabajadores sociales son "agentes de cambio social" o "promotores de la justicia social"? Comprobarlo es sencillo nuevamente con el Código de Ética:

La Sociedad Puertorriqueña, en su sistema de vivienda democrática, aspira a un orden de justicia social que alcance a todos y cada uno de sus miembros. $\mathrm{Su}$ filosofía de vida está cimentada en el valor intrínseco de la persona humana y reconoce su derecho al máximo disfrute del bienestar físico, social, intelectual, emocional y espiritual, así como su responsabilidad de participar activamente en el logro del bien común [...]. Los trabajadores sociales tienen la responsabilidad individual y colectiva de hacer realidad para todos, los principios de justicia social que constituyen la razón de ser de la profesión de Trabajo Social (Código de Ética: 1).

Aquí se demuestra que el Código es la viva representación de la herencia conservadora; aquella que naturaliza el sistema político-económico colonial. La situación es peor, ya que la presenta como "vivienda democrática" que "aspira a un orden de justicia social” y por ahí continúa alabando el colonialismo. ¿Cómo puede haber vivencia democrática en un país invadido, dominado y subordinado militarmente? Ésta es una muestra adicional de las muchas contradicciones que configuran la carrera y de las cuales hay que empezar a desprenderse.

En cuanto a lo de "luchadores por la justicia social", la verdad es que se parte de una afirmación falsa. Debe estar claro que bajo el capitalismo no puede haber (ni habrá) “justicia”, ni equidad y menos aún se puede "humanizar" o "ponerle rostro humano" porque la actual sociedad se compone e instrumenta por la desigualdad, la opresión, la explotación y la destrucción indiscriminada de los recursos naturales. En otras palabras, son el nutriente que sostienen la lógica que fundamenta la dominación de un pequeño grupo privilegiado sobre las grandes mayorías, expropiando de éstas la iniciativa, el esfuerzo, la esperanza y sobre todo, el producto de su trabajo (Marx, 1946). Y como si fuese poco, las diferencias neurálgicas del sistema de producción capitalista neoliberal se entrelazan con opresiones anteriores a éste, como el patriarcado. En definitiva, para poder alcanzar la tan mencionada "justicia social" hay que extirpar de raíz la sociedad burguesa; la lucha de clases y con ello las diferenciaciones de género.

Entonces, el Trabajo Social es una profesión que responde a situaciones fuera de su control, que no estipula y no puede manejar. Si esto es así, ¿en qué se diferencia de cualquier otro trabajador o trabajadora cualificada? Posiblemente en que es un "trabajo improductivo", lo que significa que no hay al final del día la producción de una mercancía. Sin embargo, el ejercicio se coloca en la esfera de la manutención de la fuerza de trabajo 
$\overline{\text { empleada, desempleada y sus familias. Dicho de otra manera, la }}$ materia prima es el pauperismo; los explotados y explotadas por el capitalismo; la llamada "cuestión social". ${ }^{11}$ En todo lo demás, padecen al igual que ellos de todas las reformas laborales; contratos sin seguridad laboral; centros de trabajo que en ocasiones no tienen las mínimas consideraciones de lo que debe ser un espacio decente y sano; cambios en las funciones por asuntos político-partidistas; ante la actual crisis económica son obligados a mantener turnos rotativos; no son considerados para aumentos salariales priorizando mayormente la parte punitiva; y peor aún, despidos para colocar en los puestos a "manejadores de casos", un fenómeno que se ha expandido en Puerto Rico. De hecho, posición que se desprende de la privatización de lo social y la cual mantiene bajos perfiles para ocuparla, con un salario muy por debajo de lo normal para la plaza y con muy pocos o ningunos beneficios marginales. ${ }^{12}$

En este sentido, la ecuación es sencilla; al dejar de lado la perspectiva de clase asalariada (que vende su conocimiento técnico-intelectual) se perdió la oportunidad de contrarrestar los embates del neoliberalismo, lo que por añadidura provocó que tampoco fuera defendida la tan mencionada "justicia social". Dicho eso habría que preguntarse ¿Cómo el gremio puede dar la batalla para mejorar las condiciones de vida de otros, sino tiene la fuerza para mejorar las suyas? Trágicamente, los cambios en la ${ }^{11}$ Revisar; Montaño (1998) ;Netto (1997); Iamamoto (1997); Guerra (2003). ${ }^{12}$ Sobre este fenómeno, el autor de este artículo es vivo ejemplo. De todas sus experiencias laborales solamente en dos ocasiones ha ocupado y ejercido de trabajador social, en las demás como "manejador de casos" aunque por supuesto pagando colegiación y teniendo licencia. Otro fenómeno parecido a este, sucede en el Depto. de la Familia donde muchas trabajadoras sociales ejercen puestos de técnico social. esfera económico-política golpearon fuertemente al colectivo sin ninguna respuesta que neutralizara lo que estaba sucediendo. No supieron leer la realidad, a pesar de ser las personas más insertas en la misma. ${ }^{13}$

Un dato mucho más significativo, es el hecho de que es una profesión feminizada, lo que denota que toda la agresión de precarización y tercerización de las condiciones laborales afectan directamente los derechos de la gran mayoría de mujeres que la componen. Quien está recibiendo, nuevamente, toda la agresión neoliberal, es la fuerza trabajadora femenina, lo que al final indica que el problema debe ser prioritario para el Trabajo Social en Puerto Rico por ser una cuestión de género. ${ }^{14}$

\begin{abstract}
${ }^{13}$ Aseveramos esto, porque ciertamente en distintos momentos históricos ha estado entre nuestros análisis y discursos dicho entendido. Es el caso de los y las fundadoras del Colegio de Trabajadores Sociales. En el 1940, tiempo antes de la fundación del Colegio en el preámbulo del referéndum confirmatorio, Porfirio Díaz defiende la colegiación de la siguiente manera; "Como empleados, estamos sujetos a los mismos riesgos comunes a todos lo individuos asalariados. Lo que ha probado ser efectivo para los más humildes trabajadores que se asocian en federaciones, es razonablemente justo suponerlo posiblemente efectivo para nosotros" (Díaz, 1940:13-14).

${ }^{14}$ Repasemos lo que hizo otra profesión mayoritariamente femenina en lucha por reivindicaciones salariales: la enfermería. ¿Qué hicieron estas compañeras? Simplemente, su organismo representativo se dio a la tarea de proyectar su indispensabilidad en la sociedad puertorriqueña, se organizaron, presionaron y amenazaron a las instituciones alegando que si no se ajustaban los salarios (por educación y experiencia) habría un éxodo masivo de ellas hacía Estados Unidos, cosa que había comenzado. ¿Qué lograron? Nada más y nada menos que un gran aumento para todas las profesionales de la salud. De hecho, las enfermeras prácticas (las que tienen grado asociado) tienen actualmente mayores salarios y mejores condiciones laborales que muchas trabajadoras sociales con bachillerato y posiblemente hasta con maestría. En definitiva, estos son logros que sólo pueden alcanzarse cuando hay cohesión gremial, determinación, compromiso, y entendido de clase profesional bajo condiciones deplorables de trabajo. ¿Por qué no podemos hacer lo mismo?
\end{abstract}

VOCES DESDE EL TRABAJO SOCIAL

NÚM.1 
Finalmente, hay que dejar a un lado el "clientelismo", o sea, catalogar a los individuos como "clientes". Al hacer esto, los servicios son visualizados como una mercancía y los necesitados como simples consumidores. Esta vinculación servicio-mercancía y persona-consumidor es una de las racionalizaciones más burdas del capitalismo, que entiende los ofrecimientos como costos y no como derechos adquiridos. Sobre todo, en está coyuntura neoliberal, dicha manera de entenderlo provee la oportunidad de ver en ellos una posibilidad de lucro; hecho que ya está más que comprobado. Por otro lado, cuando son nombrados de esta manera se asume la "venta" del servicio, poniendo sobre los hombros del Trabajo Social la responsabilidad de dicha reproducción ideológica. En definitiva, es debido encontrar otra forma de referirnos a los compatriotas que se acercan a reclamar sus derechos.

Como queda demostrado, hay fuerzas internas y externas; subjetivas y objetivas que inciden en eso llamado Trabajo Social puertorriqueño. Es tiempo de ver todo lo discutido gráficamente; las tensiones que presionan al Trabajo Social en su quehacer.
Figura \#1

Tensiones del ejercicio profesiona ${ }^{15}$

Tensiones de la intervención Tensiones del capital-trabajo

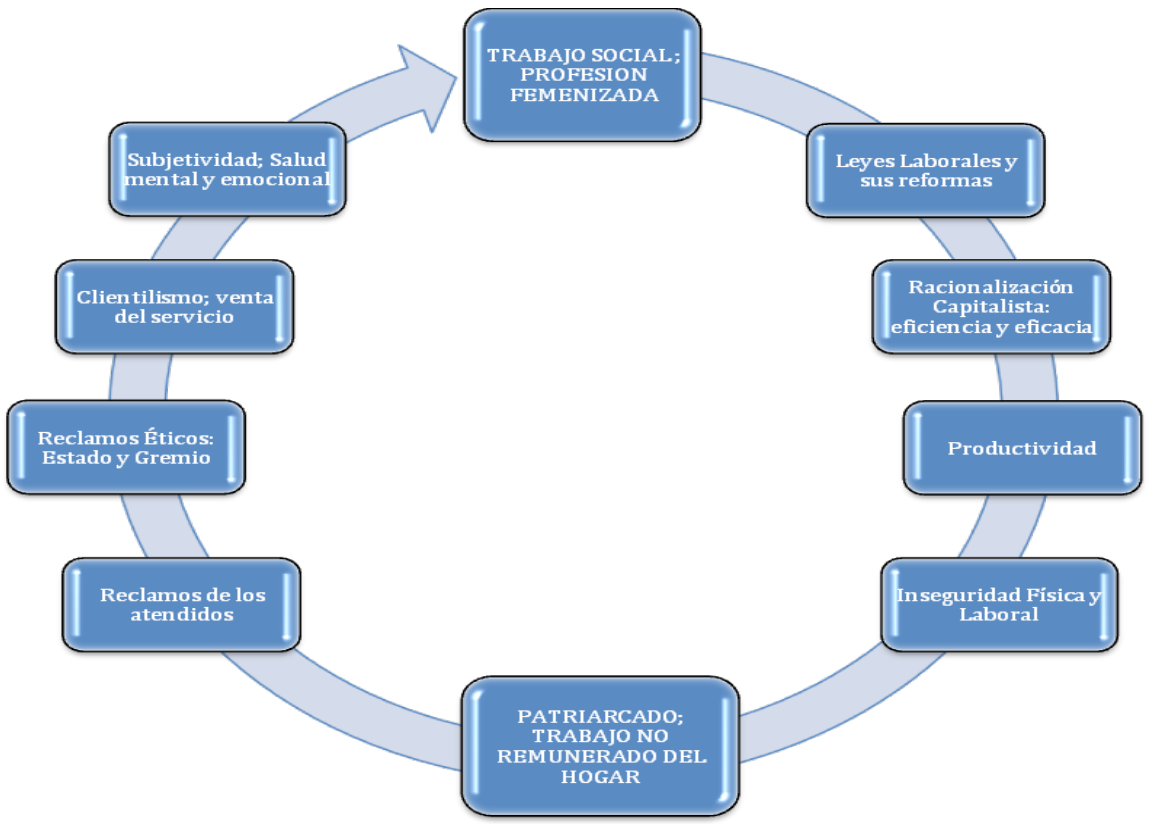

Según se presenta en la figura \#1 se puede observar hay dos grupos de tensiones. ${ }^{1614} \mathrm{Po}_{1}$ un lado, las presiones particulares de

\begin{abstract}
${ }^{15}$ La gráfica fue realizada por el autor para explicar las contradicciones del quehacer profesional en Puerto Rico, tomando en consideración los planteamientos del debate contemporáneo de Trabajo Social. Para conocer más ver el trabajo de Iamamoto (2003). Sin embargo, se incluyó un aspecto que no se ha considerado del todo dentro del mencionado debate, la cuestión de género y el patriarcado. ${ }^{16}$ Reconocemos que actualmente hay un debate naciente sobre lo privado en el Trabajo Social puertorriqueño. Aunque aceptamos dicha controversia, el grupo que vive o sobrevive en este campo es mínimo, la gran mayoría de los y las profesionales aún son asalariadas y asalariados, por lo tanto padecen lo que aquí exponemos. El conjunto que vende sus servicios de manera independiente, tiene muchas otras contradicciones, pues su posicionamiento cambia profundamente. En algunas ocasiones pasan de ser trabajadoras y trabajadores a empresarios y administradores. Utilizando dentro de sus negocios, reglamentaciones propias de la lógica empresarial.
\end{abstract}

VOCES DESDE EL TRABAJO SOCIAL

NÚM.1 
la atención e intervención en la "cuestión social". Dicho de otra manera, la carga de dar respuestas a las poblaciones con las que se trabaja: sus problemas; sus reclamos; su atención; la ejecución de la política social público-privada; las exigencias de un comportamiento ético del gremio y el Estado; junto a toda la carga emocional que ello conlleva. Por otro, las que son propias del trabajo, son aquellas situaciones que intervienen directa o indirectamente en las acciones o quehaceres diarios del "ser profesional" asalariado y que responden a la condición del capital-trabajo: la presión de las leyes laborales y sus reformas; la intervención social medida utilizando categorías cuantitativas del capitalismo como son eficacia y eficiencia, las cuales son utilizadas para establecer la productividad del empleado, y las condiciones laborales que ponen en juego la integridad física de los y las profesionales. ${ }^{17}$

Aunque de diferente naturaleza, ambas se articulan dialécticamente transformando tanto al "sujeto atendido" como al mismo trabajador social. ${ }^{18}$ Sin embargo, y como hemos venido recalcando, todas estas se complican con las características genéticas del Trabajo Social, la feminidad. Cuando la trabajadora social cumple con sus deberes laborales, comienza su segunda jornada de trabajo; todos los roles y tareas no remuneradas del hogar y

\footnotetext{
${ }^{17}$ Para ver planteamientos de esta naturaleza se recomienda revisar Díaz(1940). ${ }^{18}$ Es necesario hacer un paréntesis para explicar que hay profesiones hermanas que contienen estos tensores y características, nos referimos a la enfermería y la educación. La diferencia de éstas y nuestra profesión se define por los objetos de intervención, ambas ramas tienen bien definidos sus espacios laborales. De otra parte, las trabajadoras sociales no sólo están en dichos campos sino que se encuentras instituidas en muchos otros y en algunos con funciones represivas como parte del poder estatal. El Trabajo Social es así, la complejidad hecha vida, la cual nos ofrece una oportunidad de reconocer la realidad como totalidad.
}

la familia que asume por la imposición histórica del patriarcado. ${ }^{19}$

\section{Algunos puntos concretos para el fortalecimiento del proyecto ético-político profesional.}

En este espacio serán detalladas, puntualmente, algunas acciones que se pueden ir desarrollando como parte de ese proyecto ético-político. La naturaleza de cada uno es diferente, algunos son a corto plazo y otros se podrán lograr a largo plazo luego de un proceso de concertación y debate. Por consiguiente, y que quede meridianamente claro, no se pretende aquí decir arbitrariamente qué es lo que se debe hacer, sino lo que es posible comenzar a hacer. Debe ser aclarado algo de antemano: hay aquí señalamientos generales que atañen a toda la carrera. De igual manera, hay algunas que tienen que ver con la educación. Éstas son dirigidas a la preparación en la Universidad de Puerto Rico por ser la Escuela histórica en la Isla y la que ha determinado las demás. Aunque esto no es un limitante para que otras puedan ponerlas en práctica.

1. Primeramente hay que rectificar los errores del pasado y las injusticias que se cometieron contra grandes colegas y compatriotas. Nos referimos a dos casos que son muestra de la insensibilidad del legado conservador y colonial que nos distingue. Hablamos de las patriotas Isabel Rosado y Blanca Canales. Estamos seguros de que, efectivamente, a la colega Isabel Rosado se le retiró su licencia de Trabajo

\footnotetext{
${ }^{19}$ Aunque se puede reconocer que en cierta medida muchas de estas tensiones afectan igualmente a los hombres que componen la profesión definitivamente nunca serán internalizados y respondidos de la misma manera que las mujeres. Esto porque históricamente lo masculino se ha cimentado de ventajas, construidas y sustentadas mayormente por las religiones.
}

VOCES DESDE EL TRABAJO SOCIAL

NÚM.1 
Social y su colegiación por haber sido arrestada durante el levantamiento nacionalista del 50. En cuanto a Blanca Canales, no se ha podido confirmar pero no hay duda de que también se hizo lo mismo con ella. En este sentido, el primer paso para redefinir la profesión es impulsar una propuesta coherente y justificada para que a ambas se les devuelva, al menos, su identificación de trabajadoras sociales colegiadas en el caso de Canales, póstumamente. Esto más que ser un capricho debe ser el reconocimiento de la fortaleza, el desprendimiento, el compromiso y sobre todo, la valentía de estas dos inmensas e insignes mujeres puertorriqueñas, representantes indiscutibles de un posicionamiento éticopolítico. Este asunto tiene que ser prioridad para las recientes generaciones de trabajadoras y trabajadores sociales en Puerto Rico. Es necesario defender con ahínco este merecido reconocimiento y así comenzar con el nuevo proyecto profesional. Con dicha acción, sería reafirmado el compromiso del gremio con las mártires del Trabajo Social puertorriqueño. Blanca e Isabel deben ser, finalmente, los ejemplos y referentes hacia el futuro. Son ellas las que dictaron, y dictan el camino a seguir.

2. En relación a la educación, debe cuestionarse lo básico, el bachillerato "generalista". Hay que superar esta supuesta formación, pues reproduce todo lo discutido hasta el momento. El problema de este bachillerato "generalista" no se encuentra en el componente claustral, ni facultativo sino en el fundamento filosófico que le dio vida. Por ejemplo, sería necesario profundizar mucho más en este aspecto para construir y reestructurarlo desde los cimientos pues debe estar mayormente fundamentado en la perspectiva crítica y no en una supuesta "generalidad" de la práctica profesional. Es más, los cambios en la formación son fundamentales para crear una cepa de profesionales ética y políticamente comprometidos con dicha visión. En relación a esto, el primer paso podría ser crear cursos para discutir sobre lo ético-político y en especial el debate contemporáneo en Trabajo Social.

3. La investigación tiene que ser otro aspecto fundamental de la enseñanza. Nos han formado pensando que el Trabajo Social se nutre de todo el conocimiento generado por las Ciencias Sociales. Esto no reconoce que la profesión también aporta al mencionado cúmulo científico y la mejor prueba son las decenas de trabajos finales de graduación de la Escuela Beatriz Lasalle acumulados por más de sesenta años de investigación social. Sin embargo, dicha dimensión tiene que ser fortalecida transgrediendo la cuestión procesal de graduación. En suma, las investigaciones de grado deben ser individuales ya que las tesis grupales no tienen nada de pedagógicas. ${ }^{20}$ En cuanto a esto afirmamos, en contra de la posición generalizada, que los trabajos finales de graduación

${ }^{20}$ En la Facultad de Ciencias Sociales de la Universidad de Puerto Rico la Maestría de Trabajo Social es la única que continua promoviendo, y de cierta manera obligando, a sus estudiantes a completar investigaciones finales de graduación grupales. Esto demuestra que las demás ramas de las Ciencias Sociales han reconocido el valor incalculable de la aprehensión de dicha faceta de la educación posgraduada. De igual manera, en la mayoría de las Universidades Latinoamericanas las Tesis de Maestría son individuales. Para una mayor crítica de la investigación en la Escuela Graduada Beatriz Lasalle se recomienda la lectura de; Cabrera (2009).

VOCES DESDE EL TRABAJO SOCIAL

NÚM.1 
son proyectos de vida y cada esfuerzo realizado en las mismos son una aportación a la profesión y a nuestro país. Por lo tanto, tienen que contener un alto grado de manejo teórico y metodológico que demuestre el conocimiento de engresados y egresadas. Cosa que no se pude lograr en grupos, pues cada persona se compenetra solamente con la sección del trabajo que se le asignó, fragmentando el conocimiento y la apropiación de la pesquisa como un todo. Por otro lado, la indagación en Trabajo Social no tiene que estar circunscrita a la funcionalidad que pueda tener para la profesión, sino más bien para el conocimiento social en general. En otras palabras, si bien es cierto que la realidad laboral o de intervención nos puede ofrecer objetos de estudio, no necesariamente tiene que estar supeditada al quehacer cotidiano profesional. A nuestro entender es un enfoque muy utilitario y determinista de la investigación. Bajo un posicionamiento ético-político transparente afirmamos que: no investigamos necesariamente para la práctica profesional, lo hacemos para crear conocimiento que ayude a comprender la realidad y así poder transformarla. Finalmente, debe haber una concentración graduada en investigación que formarme una camada de profesionales especializados y especializadas en dicha materia.

4. Fomentar que los estudiantes y las estudiantes sometan a consideración de las revistas nacionales e internacionales sus trabajos de investigación. En este sentido, los bachilleratos deben crear sus propios espacios para que los y las estudiantes tengan una oportunidad de dar a conocer sus opiniones y\o pesquisas. Si por cuestiones de presupuesto no se puede lograr, al menos por el momento, sugerimos medios digitales como una página en la Internet o blogs. Este punto debe ser prioritario por varias razones. Inicialmente, ofrece la oportunidad de pasar por el temido proceso de evaluación fortalece la redacción y refuerza la confianza de cada uno de los autores. En segundo lugar, la página electrónica ofrece una gama de posibilidades que no se pueden lograr de otra manera, pues las oportunidades de publicación a través este medio son de magnitudes inimaginables. Además, es una excelente herramienta para promover, orientar e informar sobre el Trabajo Social puertorriqueño.

5. Separarnos de los organismos estadounidenses de acreditación.El Trabajo Social puertorriqueño se encuentra en momento crucial de su historia. Es hora ya de descolonizarnos, cortar de raíz la dependencia existencial que nos mantiene subordinados y subordinadas a las exigencias educativas de la nación del norte. La influencia del pragmatismo característico del "social work" estadounidense tiene que ser, por fin, superado para finalmente tomar las riendas de nuestro futuro. Es necesario que encaminemos la carrera a partir de nuestras propias metas, visiones, perspectivas y realidad cultural. ¿Por qué vienen estos personajes exógenos a decir qué ofrecer académicamente, entre muchas otras cosas? En esta misma línea, establecer mecanismos de auto-acreditación y vigorizados por la de organismos internacionales que se apeguen más a la idiosincrasia puertorriqueña. $^{21}$

6. Proponer en próximas asambleas que una parte de las becas ${ }^{21}$ Sobre esto de la acreditación estadounidense y las repercusiones en la Isla recomendamos revisar el trabajo de Garriga, L. \& Negrón, D. (2008).

VOCES DESDE EL TRABAJO SOCIAL

NúM.1 
que anualmente ofrece el Colegio esté dirigida a promover la preparación fuera de Puerto Rico, más aún si el o la estudiante desea formarse en Escuelas Graduadas en Latinoamérica. Dicha acción, proporcionará mayor retroalimentación debido a que expone a los egresados a una diferente forma de entender la profesión en todas sus dimensiones. Finalmente, a largo plazo esto es una gran inversión, pues lo invertido será retribuido al gremio como un todo.

7. Ya tenemos un grado doctoral en política social, por lo tanto hay un grupo de profesionales preparados en esta materia o en vías de lograrlo. Partiendo de esta premisa podemos inferir que ya no hay excusas para no tomar la iniciativa de proponer política pública y social. Es fundamental que la Comisión organizada en el Colegio, sobre esta materia, sea apoyada por las personas con conocimiento en este aspecto. La mencionada Comisión debe dirigir sus esfuerzos a evaluar, crear, defender y cabildear por acciones que redunden en derechos de la ciudadanía.

8. Debe ser indiscutible la defensa del servicio público. Hay que ser enfáticos y enfáticas en alertar lo nefasto de la privatización de lo social y de los servicios fundamentales. Debe ser puntual para todos y todas las trabajadoras sociales el que el Estado vuelva a asumir todo lo ya dejado en las manos de "las sin fines de lucro" por más que se entienda su buena función o ejecutoria. Tenemos que entender la privatización como un modelo práctico-ideológico del capitalismo neoliberal que se centra en la focalización y parcialización de la inversión social. Al fin y al cabo, el desenlace de dichas acciones redunda en la des-universalización de dicha inversión, ya que están dirigidas a grupos con características muy específicas y descartan a las grandes mayorías. Unido a lo anterior, es el defender sin tapujos a los trabajadores y trabajadoras del sector público de Puerto Rico.

9. Como punto final, es neurálgico denunciar el colonialismo establecido por el imperialismo estadounidense por más de cien años en el archipiélago puertorriqueño. Entendiendo que es el problema fundamental de Puerto Rico y el que transversalmente interviene con todos los demás asuntos de la cotidianidad isleña. En suma, llevar dicha denuncia a todos los foros internacionales; gremiales y políticos.

\section{A modo de conclusión}

Como se había dicho anteriormente, en Puerto Rico, los trabajadores y las trabajadoras sociales tienen que lidiar a diario, con diferentes situaciones que afectan al pueblo. La coyuntura nacional y mundial genera la necesidad de que en la Isla los y las profesionales reajusten todo lo vinculado con sus actividades, intervenciones e investigaciones. Con la debacle de los partidos políticos tradicionales, la organización de la Sociedad Civil y el creciente descontento de las y los puertorriqueños con las instituciones represivas del sistema colonial, se crean las condiciones idóneas para asumir el rol social que corresponde. Es una responsabilidad histórico-social el aceptar el reto que la propia realidad le interpone en la actualidad. Por estas razones, la importancia de lo ético-político, proyecto que proveerá la dirección que necesitamos. Por lo tanto ¡Nosotros apoyamos el proyecto ético-político! ¿Y usted? 


\section{Referencias}

Alayón, Norberto. (2005) Trabajo Social latinoamericano, A 40 años de la Reconceptualización,Buenos Aires, Argentina: Espacio Editorial

Antunes, Ricardo. (2001) ¿Adiós al trabajo? Ensayo sobre las metamorfosis y la centralidad del mundo del trabajo. Sao Paulo, Brasil: Cortez Editora.

Barroco, María. (2004) Ética y Servicio Social: Fundamentos ontológico. Sao Paulo, Brasil: Cortez Editora.

Borgiani, Elizabete \& Montaño, Carlos. (Coord.) (2000) La política social hoy. Sao Paulo, Brasil: Cortez Editora.

Borgiani,Elizabete \& Montaño,Carlos.(Coord.)(2000) Metodología y Servicio Social. Sao Paulo, Brasil: Cortez Editora.

Borgiani, Elizabete, Guerra, Yolanda. \& Montaño, Carlos. (Coord.) (2003). Servicio Social crítico. Sao Paulo, Brasil: Cortez Editora.

Burgos Ortiz, Nilsa M. (2001) Pioneras de la profesión de Trabajo Social en Puerto Rico. San juan: Publicaciones Puertorriqueñas.

Cabrera, Jesús. (2010) Capitalismo imperialista estadounidense y génesis del Trabajo Social en Puerto Rico (18981940). (Tesis de maestría no publicada). Posgrado en Trabajo Social, Universidad de Costa Rica, San José de Costa Rica.
Cabrera Cirilo,Jesús M.(2009).Perspectiva crítica delainvestigación en la Escuela Graduada Beatriz Lasalle; el caso de la génesis y desarrollo del Trabajo Social en Puerto Rico. Revista Análisis, X (1), 189-220.

Colegio de Trabajadores Sociales (1982) Código de Ética, Recuperado de http://www.ctspr.org/

Colegio de Trabajadores Sociales (2010) Cuaderno de Trabajo Proyecto Ético-Político Profesional, Reuniones Regionales, Comité de Asamblea y Proyecto Ético-Político, Documento para difusión interna y educación, Colegio de Trabajadores Sociales de Puerto Rico.

Díaz, Porfirio. (1940). La Colegiación de los Trabajadores Sociales y su Seguridad. Revista de Servicio Social, II (I),12-15.

Durkheim, Emilio. (1975) Las reglas del método sociológico. Buenos Aires, Argentina: Ediciones Orbis.

Faleiros, Vicente (2000). Las funciones de la política social en el capitalismo. En Elisabete Borgiani \& Carlos Montaño (Coord.), Metodología y Servicio Social, hoy en debate (pp. 105-120). Sao Paulo, Brasil: Cortez Editora.

Fanon, Frank. (1965) Los condenados de la tierra. México: Fondo de la Cultura Económica. 
Garriga, Lydimar. \& Negrón; Dalimar (2008). La formación neoliberal en el Trabajo Social: análisis de la filosofía, misión , metas, objetivos y contenido de los cursos medulares de la escuela graduada de Trabajo Social Beatriz Lasalle a la luz de las políticas neoliberales educativas de: la educación como mercancía y fragmentación del conocimiento y opinión de los(as) estudiantes del programa de maestría. (Tesis de maestría no publicada), Escuela Graduada de Trabajo Social Beatriz Lasalle, Universidad de Puerto Rico en Río Piedras.

Guerra, Yolanda. (2003). Instrumentalidad del proceso de trabajo y Servicio Social. En Elisabete Borgiani, Yolanda Guerra y Carlos Montaño (Coord.) (2003). Servicio Social crítico, hacia la construcción del nuevo proyecto ético-político profesional, (pp.153-170). Sao Paulo, Brasil: Cortez Editora.

Guardiola, Dagmar. (1998) Trabajo Social en Puerto Rico, ¿Asistencia, desarrollo, transformación? Río Piedras, Puerto Rico: Editorial Edil

Guardiola, Dagmar (Coord.) (2006). El Trabajo Social en el caribe hispano antillano Vol. I, el contexto regional: una perspectiva crítica. San Juan, Puerto Rico: La Editorial Universidad de Puerto Rico.

Guardiola, Dagmar (2006). El Trabajo Social en el caribe hispano antillano Vol. II, un análisis crítico de su situación: retos y perspectivas. San Juan, Puerto Rico, La Editorial Universidad de Puerto Rico.
Iamamoto, Marilda. (1997) Servicio Social y división del trabajo. Sao Paulo, Brasil: Cortez Editora. Cortez Editora.

Iamamoto, Marilda (2003) Servicio Social en la contemporaneidad. Sao Paulo, Brasil: Cortez Editora.

Iamamoto, Marilda (2000) La metodología en el Servicio Social: lineamientos para el debate. En Elisabete Borgiani \& Carlos Montaño (Coord.), Metodología y Servicio Social, hoy en debate (pp. 93-104). Sao Paulo, Brasil: Cortez Editora

Iamamoto, Marilda (2003). El debate contemporáneo del Servicio Social y la ética profesional. En Elisabete Borgiani, Yolanda Guerra \& Carlos Montaño(Coord.), Servicio Social crítico, hacia la construcción del nuevo proyecto ético-político profesional, (pp.249-270). Sao Paulo, Brasil: Cortez Editora.

Lessa, Sergio (2000). El método y su funcionamiento ontológico. En Elisabete Borgiani \& Carlos Montaño (Coord.), Metodología y Servicio Social, hoy en debate (pp.199228). Sao Paulo, Brasil: Cortez Editora.

Lessa, Sergio (2007). Trabalho e proletariado no capitalismo contemporáneo. Sao Paulo, Brasil: Cortez Editora.

Manrique, Manuel. (1982) De apóstoles a agentes de cambio, el Trabajo Social en la historia latinoamerican. Lima, Perú: Ediciones CELATS.

Marx, Carlos. (1946) El capital, tomo I. México: Fondo de Cultura Económica.

Memmi, Albert. (2001) Retrato del colonizado. Buenos Aires, Argentina: Ediciones de la Flor.

VOCES DESDE EL TRABAJO SOCIAL

NÚM.1 
Montaño, Carlos (2000). El debate metodológico de los 80\90. El enfoque ontológico versus el abordaje epistemológico. En Elisabete Borgiani \& Carlos Montaño (Coord.), Metodología y Servicio Social, hoy en debate (pp.9-34). Sao Paulo, Brasil: Cortez Editora.

Montaño, Carlos. (1998) La naturaleza del Servicio Social, un ensayo sobre su génesis su especificidad y su reproducción. Sao Paulo, Brasil: Cortez Editora.

Netto, José (1997). Capitalismo Monopolista y Servicio Social. Sao Paulo, Brasil: Cortez Editora.

Netto, José (2000). Método y teoría en las diferentes matrices del Servicio Social. En Elisabete Borgiani \& Carlos Montaño (Coord.), Metodología y Servicio Social, hoy en debate (pp.51-92). Sao Paulo, Brasil: Cortez Editora.

Netto, José (2003). El Servicio Social y la tradición marxista. En Elisabete Borgiani, Yolanda Guerra \& Carlos Montaño (Coord.), Servicio Social crítico, hacia la construcción del nuevo proyecto ético-político profesional, (pp.153-170). Sao Paulo, Brasil: Cortez Editora.

Pereira, Potayra. (2002) Necesidades humanas, para una crítica a los patrones mínimos de sobrevivencia. Sao Paulo, Brasil: Cortez Editora.

Pereira, Potayra (2003). Perspectivas teóricas sobre a questao social no Servico Social. Revista Temporalis, IV (7), 112122. Porto Alegre, Brasil: Associação Brasileira de Ensino e Pesquisa em Serviço Social-ABEPSS.
Quiroga, Consuelo (2000). Invasión positivista en el marxismo: el caso de la enseñanza de la metodología en el Servicio Social. En Elisabete Borgiani \& Carlos Montaño (Coord.), Metodología y Servicio Social, hoy en debate (pp. 121170). Sao Paulo, Brasil: Cortez Editora.

Rojas, Rosa (2001). Métodos para la investigación social una proposición dialéctica. México: Plaza y Valdés Editores.

Romeral, R del. (1904) La cuestión social y Puerto Rico, San Juan, Puerto Rico, en microficha.

Seda, Raquel (1990). El desarrollo del Trabajo Social en Puerto Rico: una visión critica. Ponencia presentada en al Quincuagésima Primera Asamblea Anual del Colegio de Trabajadores Sociales de Puerto Rico, celebrada del 26 de octubre de 1990, San Juan, Puerto Rico.

\section{Entrevista citada en el documento}

Cabrera, J. (2009) Entrevista realizada a la Dra. Raquel Seda, el domingo 1 de marzo de 2009, en Mayagüez Puerto Rico. 